University of Texas at El Paso

ScholarWorks@UTEP

$5-2000$

\title{
Fuzzy (Granular) Levels of Quality, with Applications to Data Mining and to Structural Integrity of Aerospace Structures
}

Roberto A. Osegueda

The University of Texas at El Paso

Carlos Ferregut

The University of Texas at El Paso

Vladik Kreinovich

The University of Texas at El Paso, vladik@utep.edu

Seelam Seetharami

Harry Schulte

The University of Texas at El Paso

Follow this and additional works at: https://scholarworks.utep.edu/cs_techrep

Part of the Computer Engineering Commons

Comments:

UTEP-CS-00-22.

Published in Proceedings of the 19th International Conference of the North American Fuzzy Information Society NAFIPS'2000, Atlanta, Georgia, July 13-15, 2000, pp. 348-352.

\section{Recommended Citation}

Osegueda, Roberto A.; Ferregut, Carlos; Kreinovich, Vladik; Seetharami, Seelam; and Schulte, Harry, "Fuzzy (Granular) Levels of Quality, with Applications to Data Mining and to Structural Integrity of Aerospace Structures" (2000). Departmental Technical Reports (CS). 479.

https://scholarworks.utep.edu/cs_techrep/479

This Article is brought to you for free and open access by the Computer Science at ScholarWorks@UTEP. It has been accepted for inclusion in Departmental Technical Reports (CS) by an authorized administrator of ScholarWorks@UTEP.For more information, please contact Iweber@utep.edu. 


\title{
Fuzzy (Granular) Levels of Quality, With Applications to Data Mining and to Structural Integrity of Aerospace Structures
}

\author{
Roberto A. Osegueda ${ }^{1}$, Carlos Ferregut ${ }^{1}$, \\ Vladik Kreinovich $^{1}$, Seelam Seetharami ${ }^{1}$, \\ and Harry Schulte ${ }^{2}$ \\ ${ }^{1}$ FAST Center for Structural \\ Integrity of Aerospace Structures \\ and \\ ${ }^{2}$ Digital Media Center \\ University of Texas at El Paso \\ El Paso, TX 79968, USA \\ contact email vladik@cs.utep.edu
}

\begin{abstract}
Experts usually describe quality by using words from natural language such as "perfect", "good", etc. In this paper, we deduce natural numerical values corresponding to these words, and show that these values explain empirical dependencies uncovered in data mining and in the analysis of structural integrity of aerospace structures.
\end{abstract}

\section{Formulation of the Problem}

In mathematical descriptions, quality is characterized by a numerical value of an appropriately chosen objective function.

In real-life, however, to describe quality, we use words such as "perfect", "good", etc.

We therefore need to relate numerical values with words describing quality.

- If we already have a numerical value, then fuzzy logic provides us with a reasonable technique for translating this numerical value into words.

- Often, we face the opposite problem: we have an expert's estimate of quality in terms of words, and we must translate this estimate into numbers so that we will be able to combine this quality information with other knowledge which is already given in numerical form.
Of course, we can always use the general knowledge elicitation techniques of fuzzy logic. However, since quality words appear in various environments, it is desirable to develop general environment-independent techniques for formalizing quality words.

\section{Historically First Result: Formalization of the Word "Perfect"}

\subsection{Main Idea}

In this paper, we exploit the idea which was first developed in [8] (see also [9]). This idea is as follows:

"perfect" means that a further increase in intensity would result in an opposite effect.

\subsection{Towards Formalization of This Idea}

In [9], this idea is used to describe the perfect time allocated for cooperative learning. Cooperation is advantageous, but some learning is best done individually. What portion $x$ of class time should be allocated to cooperative learning and what portion to individual learning? If we start with 0 portion $x=0$ (no collaboration at all), and gradually increase it (i.e., add some cooperation), we will make the learning more efficient. However, after a certain value $x$, we will get a decrease in efficiency.

The optimal (perfect) portion $p$ allocated for cooperative learning can be described by the following condition: 
further increase in this degree leads to an opposite effect. We can rewrite this condition as follows:

$$
\text { very } p=\operatorname{not} p \text {. }
$$

To formalize "very" and "not", it is natural to use fuzzy logic (see, e.g., [6, 12]):

- "very" $x$ is typically interpreted as $x \cdot x$, and

- "not" $x$ is usually interpreted as $1-x$.

Comment. The interpretation of "very" as $x \cdot x$, originally proposed by in the pioneer paper [15], was experimentally confirmed in [7].

\subsection{Formalization and the Resulting Numerical Value of "Perfect"}

If we use these interpretations in the above formula, we get the equation $p \cdot p=1-p$, whose only solution on the interval $[0,1]$ is the golden proportion number

$$
p=\frac{\sqrt{5}-1}{2}=0.618 \ldots
$$

\subsection{Confirmation of This Numerical Value}

The validity of the above analysis is confirmed both:

- by the general fact that the golden proportion is known to be esthetically pleasing, and also

- by the experimental results in learning according to which the optimal portion of cooperative education is around $60 \%[4,5,9]$.

\section{Natural Next Result: Formalization of the Word "Good"}

\subsection{Idea}

While formalizing the notion of "perfect", we also discussed how to naturally formalize the word "very". Now, to find the natural numerical equivalent $g$ of the word "good", we can relate this word to the words whose formalization we already know. A natural common-sense relation is that "perfect" can be interpreted as "very good".

\subsection{Formalization and the Resulting Numerical Value of "Good"}

Since we know that "very" $x$ means $x \cdot x$, we thus conclude that $g \cdot g=p=0.618 \ldots$, i.e., that $g=\sqrt{p}=$ 0.77 ... (i.e., $g$ is approximately equal to 0.8 ).

\subsection{Confirmation of This Numerical Value}

This value is also in good accordance with common sense, namely, with the 20-80 "Pareto" law, according to which:

- $20 \%$ of the people drink $80 \%$ of the beer,

- $20 \%$ of the researchers write $80 \%$ of all papers etc.

All these "20-80" descriptions can be reformulated, in common-sense terms, by statements like "people who are not very good to their health drink a good amount of beer', etc.

\section{Applications to Data Mining}

\subsection{First Application: Idea}

In data mining, we take the data (e.g., a big sales database), and try to find new rules in the seemingly ruleless data. An interesting and somewhat unexpected practical result of using data mining techniques is that a good number of these automatically discovered rules turns out to be already known by experts.

\subsection{First Application: Conclusion}

If we use the above formalization of the word "good", then we conclude that about $80 \%$ of the automatically discovered rules are already known to experts.

\subsection{First Application: Experimental Confirma- tion}

The above conclusion is in good accordance with the experimental fact that from each 25 rules typically discovered by a system, approximately 20 are already known (see, e.g., [11]).

\subsection{Second Application: Idea}

The above experimental fact does not mean that the remaining 5 rules are immediately accepted by the experts: these "rules" are often accidental coincidences which happen to occur in this particular batch of data but which will probably not be confirmed by the follow-up data.

This is one of the reasons why, in spite of a lot of computer efforts, there are not so many success stories in data mining as one could expect, and the same impressive stories move from one popular article to the other. 
For a rule to be accepted by experts, it has to appear consistently; at least, it has to appear again in the new batch of data.

A common sense description of this phenomenon is that a good number of new rules do not appear again.

\subsection{Second Application: Conclusion}

If we use the above interpretation of the word "good", we conclude that only $20 \%$ of the new rules are confirmed. Since new rules form $20 \%$ of the total number of rules uncovered by a data mining software, we can thus conclude that only $20 \% \cdot 20 \%=4 \%$ of the rules discovered by this software turns out to be real new rules, i.e., rules which are: not only previously unknown, but also acceptable to experts (and thus useful in future applications).

\subsection{Second Application: Experimental Confir- mation}

This number is again in good accordance with the statistics given in [11] who say that, on average, our of every 25 un-covered rules, only one turn out to be a useful new rule.

\subsection{Data Mining: Conclusion}

Thus, our formalization of quality words explains the empirical phenomena from data mining described in [11].

\section{Applications to Structural Integrity of Aerospace Structures}

\subsection{Formulation of the Problem}

As a second case study, we considered the nondestructive analysis of structural integrity of aerospace structures. This is an important practical problem, in which, due to a large uncertainty, methods of soft computing have been very useful (see, e.g., [2]). These methods are not perfect, they can erroneously overlook some faults. These errors come from two sources:

- Part of these errors are caused by the uncertainty of the problem: we are restricting ourselves to nondestructive testing, which is inevitably imprecise for inner faults).

- Part of these errors are caused by the imperfections of the methods.

To avoid a useless perfecting of an imperfect situation, it is desirable to have a rule-of-thumb expectation of how many errors we should expect; then, if a processing method reaches this error rate, it means that we have achieved our goal, and further perfecting is rather useless.

\subsection{First Application: Idea}

The expert estimates of these rates use the quality words: that a good number of faults can be thus identified.

\subsection{First Application: Conclusion}

If we use the above interpretation of the word "good", then we can conclude that we should expect up to $77 \%$ of the faults to be identifiable by these techniques.

\subsection{First Application: Experimental Confirma- tion}

Current non-destructive estimation techniques have almost reached that level: they un-cover more than $70 \%$ of the faults (see, e.g., [1, 10], and references therein).

\subsection{Additional Conclusion: Only Data Fusion Can Drastically Decrease the Error Rate}

At first glance, this result does not seem to be very encouraging: even when we perfect our methods, they will still miss every fifth fault. However, this result is not as bad as it seems:

- This estimate is about using a single method.

- However, there exist several different soft computing techniques, each of which has its own problems and successes.

It is therefore natural to use data fusion, i.e., to combine several different techniques together.

\subsection{Second Application: Idea}

What is the expected error rate for data fusion methods?

Under the combination of two methods, we expect that a good number of faults overlooked by one of the methods will be located by the second one.

\subsection{Second Application: Conclusion}

If we use the above formalization of the word "good", then, similarly to data mining, we can make the following conclusion: With two methods, we expect to overlook only $20 \% \cdot 20 \%=4 \%$ of the faults - i.e., correctly detect $96 \%$ of them. 


\subsection{Second Application: Experimental Confir- mation}

General conclusion. Our preliminary results have shown that while, say, fine-tuning fuzzy methods does not lead to a serious performance improvement, data fusion can indeed drastically improve the performance; see, e.g., [13].

Let us describe the details of our experiment is some detail.

Techniques used. To test different fusion techniques, we took a plate with several known faults of different sizes, and applied different non-destructive techniques (such as pulse echo, Eddy current, and resonance) to this plate. For each of these techniques, we got, for each point on the plate, the corresponding signal values.

General idea of non-destructive testing. The general idea of non-destructive fault detection is as follows:

- If one of these techniques leads to the unusual signal value (very different from the normal values), then a fault is probably located at this point.

- The larger the difference, the larger is the fault.

How to implement this idea: case when we know the pre-flight inspection results. Let's show how this idea can be formalized. First, we must detect the faults. In accordance with the above idea, in order to detect a fault, we must know the normal values of the signal and the allowable deviation from these normal values.

In some cases, we have the results of the initial (preflight) inspection, in which no faults were found. In such cases, for each type of the signal, we can compute:

- the (arithmetic) average $a$ of all the values, and

- the mean square (standard) deviation $\sigma$ of the signal $x$ from its average $a$.

In accordance with the standard statistical techniques (see, e.g., [14]), we can then consider values $x$ which are outside of the "three sigma" interval $[a-3 \sigma, a+3 \sigma]$ as possible faults.

Problem with the above straightforward implementation. The problem with this (seemingly straightforward) approach is that for all the above methods, unusual values occur not only at the fault locations, but also at the edges of the plate.

The relation between faults and edges is very natural, since, e.g., a typical fault is a hole in the plate, i.e., an extra edge.

As a result of this relation, the computation of the the average $a$ and of the standard deviation $\sigma$ involve the edge points, in which the value of the signal is very different from the expected normal value. Hence, the computed $\sigma$ is much larger than for the normal points. As a result, some true faults - which would have been detected by a correct (smaller) $\sigma-$ go undetected when we use this larger value.

Solution to this problem: case when we know the preflight inspection results. If we know the results of the initial inspection, then we can ignore the edges when computing the average and $\sigma$, and get correct results.

This solution does not work in other cases. In many practical situations, we do not have these initial inspection results (e.g., if what we are doing in exactly this preflight inspection). In such situations, we must estimate the values $a$ and $\sigma$ based on the plate with faults.

In this case, even if we eliminate the edges, we may still have faults, and the signals corresponding to these faults "spoil" the values $a$ and $\sigma$ and prevent us from detecting all the faults.

A new solution to the above problem: idea. To eliminate all the faults, we can:

- first, mark the worst faults (which can be detected even when we use the enlarged $\sigma$ );

- then, re-calculate $a$ and $\sigma$ by omitting known faults,

- check for new faults by using these better estimates for $a$ and $\sigma$,

and continue these iterations until all the faults are uncovered.

A new solution to the above problem: algorithm. As a result, we arrive at the following iterative algorithm. At each step $s=1,2, \ldots$ of this algorithm:

- new approximations $a^{(s)}$ and $\sigma^{(s)}$ are computed for the desired values of $a$ and $\sigma$; and

- some points on the plate are marked as either edge points or possible fault locations.

In the beginning, we do not know where the faults are, so no points are marked. Each step $s$ consists of the following two operations:

- first, we compute the average $a^{(s)}$ and the standard deviation $\sigma^{(s)}$ based on all un-marked points of the plate;

- then, we check each un-marked point, and mark all the points for which $\left|x-a^{(s)}\right| \geq 3 \sigma^{(s)}$.

The process stops when the subsequent values of $a^{(s)}$ and $\sigma^{(s)}$ become close to each other (this usually happens on the $3 \mathrm{rd}$ or 4 th steps). The corresponding final values of 
$a^{(s)}$ and $\sigma^{(s)}$ are then taken as the desired values of $a$ and $\sigma$.

Resulting data fusion: preparation. For each marked point, we then estimate the deviation $d=(x-a) / \sigma$. Based on the plate with known faults, we calibrate the dependence $d(r)$ of the deviation $d$ on the size $r$ of the fault; then, based on the observed deviation $d$, we can estimate its size $r$ by solving the equation $d(r)=d$.

Data fusion itself. From several different testing signals, we get several estimates from the size and location of the fault. We can then combine these estimates, e.g., by using standard statistical averaging [14].

\subsection{Structural Integrity: Conclusion}

The above optimistic estimate encourages us to put all the efforts not into perfecting one of the known techniques, but rather into combining several existing ones into a single fused approach.

\section{Acknowledgment}

This work was supported in part by NASA under cooperative agreement NCC5-209, by NSF grants No. DUE-9750858 and CDA-9522207, by the United Space Alliance, grant No. NAS 9-20000 (PWO C0C67713A6), by the Future Aerospace Science and Technology Program (FAST) Center for Structural Integrity of Aerospace Systems, effort sponsored by the Air Force Office of Scientific Research, Air Force Materiel Command, USAF, under grant number F49620-95-1-0518, and by the National Security Agency under Grant No. MDA904-98-10561 .

\section{References}

[1] C. Ferregut, R. A. Osegueda, Y. Mendoza, V. Kreinovich, and T. J. Ross, "Aircraft Integrity and Reliability", In: J. Booker, J. Parkinson, and T. R. Ross (eds.), Combined Fuzzy Logic and Probability Applications, SIAM, 2000 (to appear).

[2] C. Ferregut, R. A. Osegueda, and A. Nuñez (eds.), Proceedings of the International Workshop on Intelligent NDE Sciences for Aging and Futuristic Aircraft, El Paso, TX, September 30-October 2, 1997.

[3] X. E. Gros, NDT Data Fusion, J. Wiley, London, 1997.

[4] D. W. Johnson, R. T. Johnson, and E. Holubec, Circles of learning: cooperation in the classroom, Interactive Book Co., Edina, MN, 1990.
[5] D. W. Johnson, R. T. Johnson, and K. A. Smith, Active learning: cooperation in the college classroom, Interactive Book Co., Edina, MN, 1991.

[6] G. Klir and B. Yuan, Fuzzy sets and fuzzy logic: theory and applications, Prentice Hall, Upper Saddle River, NJ, 1995.

[7] M. Kochen and A. N. Badre, "On the precision of adjectives which denote fuzzy sets", J. Cybern., 1976, Vol. 4, No. 1, pp. 49-59.

[8] M. Koshelev, "Fuzzy logic explains the golden proportion", International Journal of Intelligent Systems, 1997, Vol. 12, pp. 415-417.

[9] V. Kreinovich, E. Johnson-Holubec, L. K. Reznik, and M. Koshelev, "Cooperative learning is better: explanation using dynamical systems, fuzzy logic, and geometric symmetries", In: Nguyen Hoang Phuong and A. Ohsato (eds.), Proceedings of the Vietnam-Japan Bilateral Symposium on Fuzzy Systems and Applications VJFUZZY'98, HaLong Bay, Vietnam, 30th September-2nd October, 1998, pp. 154-160.

[10] M. Krishna, V. Kreinovich, and R. A. Osegueda, "Fuzzy Logic in Non-Destructive Testing of Aerospace Structures", Proceedings of the 1999 IEEE Midwest Symposium on Circuits and Systems, Las Cruces, New Mexico, August 8-11, 1999 (to appear).

[11] R. Kruse, C. Borgelt, and D. Nauck, "Fuzzy data analysis: challenges and perspectives", partly published in: Proceedings of the 8th IEEE International Conference on Fuzzy Systems (FUZZIEEE'99), Seoul, Korea, August 22-25, 1999, Vol. 3, pp. 1211-1216.

[12] H. T. Nguyen and E. A. Walker, First course in fuzzy logic, CRC Press, Boca Raton, FL, 1999.

[13] R. A. Osegueda, A. Revilla, L. Pereyra, and O. Moguel, "Fusion of modal strain energy differences for localization of damage", In: A. K. Mal (ed.), Nondestructive Evaluation of Aging Aircraft, Airports, and Aerospace Hardware III, Proceedings of SPIE, 1999, Vol. 3586, Paper 3586-28.

[14] H. M. Wadsworth, Jr. (editor), Handbook of statistical methods for engineers and scientists, McGrawHill Publishing Co., N.Y., 1990.

[15] L. A. Zadeh, "Outline of a new approach to the analysis of complex systems and decision processes", IEEE Transactions on Systems, Man and Cybernetics, 1973, Vol. 3, pp. 28-44. 